\title{
EDITORIAL \\ Global epidemiology of infections due to Shigella, Salmonella serotype Typhi, and enterotoxigenic Escherichia coli
}

\author{
M. A. MILLER ${ }^{1 *}$, J. SENTZ ${ }^{1}$, M. A. RABAA ${ }^{1}$ AND E. D. MINTZ ${ }^{2}$ \\ ${ }^{1}$ Division of International Epidemiology and Population Studies, Fogarty International Center, \\ National Institutes of Health, Bethesda, MD, USA \\ ${ }^{2}$ Enteric Diseases Epidemiology Branch, National Center for Zoonotic, Vectorborne, and Enteric Diseases, \\ Centers for Disease Control and Prevention, Atlanta, GA, USA
}

(Accepted 15 January 2008)

Enteric infections are a leading cause of disease and death in young children, particularly in the developing world [1]. In 1990, the World Health Organization (WHO) Global Burden of Disease estimated that diarrhoea contributed to 2.5 million deaths in children aged $<5$ years, representing $19 \cdot 4 \%$ of total mortality in that age group [2]. Preliminary estimates from WHO for 2000 indicate that $1 \cdot 6-2 \cdot 1$ million deaths occurred in children aged $<5$ years during that year [3-5]. While the declines are promising, and suggest that progress was made in the intervening decade, the confidence intervals surrounding these estimates are wide, especially at the national and subnational levels, and pathogen-specific morbidity and mortality are poorly characterized. Although mortality rates due to diarrhoea have decreased globally, diarrhoea morbidity rates have not followed the same trend, especially in poor regions experiencing rapid population growth, where morbidity due to diarrhoea may actually have increased in recent years [6]. Furthermore, diarrhoea morbidity and mortality are probably greatest in areas where resources for surveillance, diagnosis, prevention, and treatment are most scarce, areas that are often under-represented in national estimates of diarrhoea incidence that are based on data from health facilities.

New tools for the prevention and treatment of enteric infections have become available in recent years. These include pathogen-specific interventions such as new vaccines and improved diagnostics with

\footnotetext{
* Author for correspondence: M. A. Miller, M.D., 16 Center Drive, Bethesda, MD, USA, 20892.

(Email: millemar@mail.nih.gov)
}

which to target treatment [7], as well as interventions that may affect a variety of pathogens, such as new approaches to household water treatment and storage [8], new strategies for the promotion of sanitation [9] and hand hygiene [10], nutritional supplementation [11], integrated case management with low-osmolarity oral rehydration solution [12], and zinc supplementation [13]. However, little is known about the relative or combined impact of these interventions on enteric infections in general, or about their pathogen-specific effectiveness, in terms of health and economic benefits. The rational allocation of resources for treatment and prevention by policy-makers and funders requires accurate information on the pathogen-specific burden of disease and death, and on the cost-effectiveness of available interventions on pathogen-specific morbidity and mortality.

Financial and human resource constraints have limited the opportunities to perform accurate assessments of the disease burden caused by specific enteric pathogens and of the effectiveness of various interventions, especially in the developing world. Estimates of pathogen-specific disease burden are rarely population-based, inclusive of all age groups, or designed to capture all relevant health outcomes. Public health policy is frequently based on extrapolation of findings from limited controlled trials and may not accurately reflect localized variations, which influence the more relevant measure of practical effectiveness. For example, age-specific data are required to determine the potential preventable fraction by a vaccine given during infancy. Intervention studies often do not have the power to measure 
impacts on illnesses caused by specific pathogens, or on specific sequelae of enteric infections such as malnutrition. In addition, assessments of specific interventions are frequently constrained by the potential bias of short-term perspectives and advocacy, and may not reflect longer term biological and ecological factors or changes resulting from economic development.

To identify more clearly the gaps in our understanding of the epidemiology of enteric infections in the developing world, with support from the Bill and Melinda Gates Foundation, we conducted extensive literature reviews of three aetiological agents, Shigellae, Salmonella enterica serotype Typhi, and enterotoxigenic Escherichia coli. For each, we also reviewed data on specific interventions and diagnostic tools that can be used to better assess efforts to reduce mortality and morbidity associated with these pathogens.

For each pathogen, we searched the PubMed database using the same set of keywords and certain pathogen-specific terms. We restricted the results to studies conducted in low- and medium-income countries, and published between 1984 and 2005. Population-based studies with culture confirmation of cases were considered primary data sources; when these were lacking, hospital-based studies were considered.

This series of reviews can serve as a guide to our current knowledge of the epidemiology of these three major bacterial enteric pathogens [14-16]. A fourth review paper on infections due to Vibrio cholerae has been published elsewhere [17]. The knowledge gaps identified can serve as a roadmap of research activities for those who are interested in funding efforts to better control these diseases with targeted or general interventions.

Of particular note, our findings indicate that most, if not all, of the bacterial enteric infections are environmentally determined and respond to secular changes in hygiene and sanitation. Studies that were conducted in populations 20 years ago and indicate a high incidence of infection cannot be extrapolated to the same populations today, as they may have undergone substantial development. For example, the 14500 deaths from diarrhoea in children aged $<5$ years in Mexico in 1990 was reduced to 5095 by 1995 , and has been reduced even further in recent years, with a change from summertime deaths, thought to result from bacterial infections, to wintertime deaths, presumably caused by rotavirus [18, 19]. Municipal water and sanitation services greatly contributed to the reduction that occurred during this period [20, 21].

While a significant portion of worldwide diarrhoeal disease mortality can be attributed to the pathogens reviewed in these articles, they are among a diverse group of pathogens responsible for global morbidity resulting from enteric infections. Among these, G. lamblia, C. parvum, and enteroaggregative E. coli (EAEC) have received increasing attention in recent years because of their effects on child development [22-27]. Development of new diagnostics and investigation of host and microbial genetic factors will further elucidate the complex relationships between enteric pathogens, diarrhoeal illnesses, and child development.

The papers in this series provide background on the demographic and geographic distributions and knowledge gaps of the epidemiology of three major bacterial enteric infections. We hope that these reviews will help to optimize the implementation of targeted approaches to disease control.

\section{ACKNOWLEDGEMENTS}

This work was supported in part by the U.S. National Institutes of Health Fogarty International Center and by grant number 32143 from the Bill and Melinda Gates Foundation 'Assessment of diarrhoea disease burden and public health programs to control diarrhoea in the Asian subcontinent and Africa'.

\section{DECLARATION OF INTEREST}

None.

\section{REFERENCES}

1. Black RE, Morris SS, Bryce J. Where and why are 10 million children dying every year? Lancet 2003; 361: 2226-2234.

2. Murray CJL, Lopez AD. The Global Burden of Disease, A Comprehensive Assessment of Mortality and Disability from Diseases, Injuries, and Risk Factors in 1990 and Projected to 2020. Harvard: Harvard University Press, 1996.

3. Boschi-Pinto C, Tomaskovic L. Deaths from diarrhoeal diseases among children under five years of age in the developing world: a review. Bulletin of the World Health Organization (in press).

4. Parashar UD, et al. Global illness and deaths caused by rotavirus disease in children. Emerging Infectious Diseases 2003; 9: 565-572. 
5. Bryce $\mathbf{J}$, et al. Can the world afford to save the lives of 6 million children each year? Lancet 2005; 365: 2193-2200.

6. Guerrant RL, et al. Magnitude and impact of diarrheal diseases. Archives of Medical Research 2002; 33: 351-355.

7. Ricci KA, et al. Reducing stunting among children: the potential contribution of diagnostics. Nature 2006; 444 (Suppl. 1): 29-38.

8. Lantagne DS, Quick R, Mintz ED. Household water treatment and safe storage options in developing countries: a review of current implementation practices. In: Parker M, Williams A, Youngblood C, eds. Water Stories: Expanding Opportunities in Small-scale Water and Sanitation Projects. Washington, DC, USA: Woodrow Wilson International Center for Scholars, Environmental Change and Security Program, 2007, pp. 17-38.

9. Barreto ML, et al. Effect of city-wide sanitation programme on reduction in rate of childhood diarrhoea in northeast Brazil: assessment by two cohort studies. Lancet 2007 ; 370: 1622-1628.

10. Luby SP, et al. Effect of handwashing on child health: a randomised controlled trial. Lancet 2005; 366: 225-233.

11. Aggarwal R, Sentz J, Miller MA. Role of zinc administration in prevention of childhood diarrhea and respiratory illnesses: a meta-analysis. Pediatrics 2007; 119: 1120-1130.

12. Benguigui Y, Stein F. Integrated management of childhood illness: an emphasis on the management of infectious diseases. Seminars in Pediatric Infectious Diseases 2006; 17: 80 .

13. WHO. Technical Update of the Guidelines on Integrated Management of Childhood Illness (IMCI). Geneva: World Health Organization, 2005.

14. Crump JA, et al. Part I. Analysis of data gaps pertaining to Salmonella enterica serotype Typhi infections in low and medium human development index countries, 1984-2005. Epidemiology and Infection. Published online: 9 August 2007. doi:10.1017/S0950268807009338.

15. Ram PK, et al. Part II. Analysis of data gaps pertaining to Shigella infections in low and medium human development index countries, 1984-2005. Epidemiology and Infection. Published online: 9 August 2007. doi:10.1017/S0950268807009351.

16. Gupta SK, et al. Part III. Analysis of data gaps pertaining to enterotoxigenic Escherichia coli infections in low and medium human development index countries, 1984-2005. Epidemiology and Infection. Published online: 9 August 2007. doi:10.1017/ S095026880700934X.

17. Griffith DC, Kelly-Hope LA, Miller MA. Review of reported cholera outbreaks worldwide, 1995-2005. American Journal of Tropical Medicine and Hygiene 2006; 75: 973-977.

18. Villa S, et al. Seasonal diarrhoeal mortality among Mexican children. Bulletin of the World Health Organization 1999; 77: 375-380.

19. Velázquez FR, et al. Diarrhea morbidity and mortality in Mexican children: impact of rotavirus disease. Pediatric Infectious Disease Journal 2004; 23: S149-155.

20. Gutiérrez G, et al. Impact of oral rehydration and selected public health interventions on reduction of mortality from childhood diarrhoeal diseases in Mexico. Bulletin of the World Health Organization 1996; 74: 189-197.

21. Sepulveda J, Valdespino JL, Garcia-Garcia L. Cholera in Mexico: The paradoxical benefits of the last pandemic. International Journal of Infectious Diseases 2006; 10: 4-13.

22. Guerrant RL, et al. Diarrhea as a cause and an effect of malnutrition: diarrhea prevents catch-up growth and malnutrition increases diarrhea frequency and duration. American Journal of Tropical Medicine and Hygiene 1992; 47: 28-35.

23. Schorling JB. A prospective study of persistent diarrhea among children in an urban Brazilian slum. Patterns of occurrence and etiologic agents. American Journal of Epidemiology 1990; 132: 144-156.

24. McAuliffe JF. Prolonged and recurring diarrhea in the northeast of Brazil: examination of cases from a community-based study. Journal of Pediatric Gastroenterology and Nutrition 1986; 5: 902-906.

25. Checkley W, et al. Effects of acute diarrhea on linear growth in Peruvian children. American Journal of Epidemiology 2003; 157: 166-175.

26. Lima AA. Persistent diarrhea signals a critical period of increased diarrhea burdens and nutritional shortfalls: a prospective cohort study among children in northeastern Brazil. Journal of Infectious Diseases 2000; 181: 1643-1651.

27. Steiner TS, et al. Enteroaggregative Escherichia coli produce intestinal inflammation and growth impairment and cause interleukin- 8 release from intestinal epithelial cells. Journal of Infectious Diseases 1998; 177: 88-96. 